\title{
Polarimetric Method to Discriminate the Drops on Sizes within the Resolution Volume
}

\author{
Yuliya Averyanova \\ Air Navigation Systems Department \\ National Aviation University \\ Kiev, Ukraine \\ ayua@nau.edu.ua
}

\author{
Felix Yanovsky \\ Electronics Department \\ National Aviation University \\ Kiev, Ukraine
}

\begin{abstract}
In this paper we consider possibilities of radar polarimetry to solve different meteorological tasks including identification of atmospheric pfenomena microstructure and hydrometeor orientation, hydrometeors size measurement, wind related phenomena estimation. The potentials and restrictions of radar polarimetry are discussed. The approach that allows to increase informativity of polarization measuremens by distinguishing hydrometeors with similar polarization properties inside the reflecting volume and estimating their differential impact onto polarization properties of reflected signal is presented. The overvew of the polarimetric system with multiple receiving antenna system for aproach realization is done. The results are important for different practical applications of radar meteorology including drop size distribution estimate, rainfall intensity estimate, wind speed measurement, turbulence and strong wind shear detection.
\end{abstract}

Index Terms - Radar meteorology; polarimetry; scattering; scattering volume; drops

\section{INTRODUCTION}

The development of meteorlogical radars since the middle of $20^{\text {th }}$ century leads to the appearance and wide operation of powerful tools for solving many question related to aviation, meteorology, agriculture, hydrometeorology and other fields. Nowadays the systems of different ranges (radars, lidars, and sodars) are used for meteorology related tasks as well as those that retrieve information from different parameters of electromagnetic oscillations (Doppler and Polarimetric radars). The most promising instrument for obtaining information about atmospheric state and processes are combined Doppler and Polarimetric Radars. Their advantages and possibilities are shown in [1-6].

The polarimetry in modern polarimetris and DopplerPolarimetric radars is used for hydrometeor identification mostly. But the fact that radar signal reflectors change their shape and orientation under the wind related phenomena influence is promising for operation with polarimetry for wind related phenomena detection and estimation. Some approaches that illustrate this possibility are presented in [7-10]. These approaches are based on the behavior peculiarities of different liquid hydrometeors under the wind related phenomena influence that change their shape and orientation [11,12]. Thus, it can be useful to evaluate the external influence character and its intensity through the change of drop orientation, shape variations and vibration of drops that, in turn, impact onto the polarimetric features of the reflected radar signal.

In real situation peculiarities of drop behavior caused by wind related phenomena can be veiled beyond the averaged estimates of reflections from hydrometeors assemble. Therefore, distinguishing hydrometeors with similar polarization properties inside the reflecting volume and estimating their differential impact into the polarization properties of reflected signal are important tasks for practical implementation of polarimetric methods for wind related phenomena detection and estimate.

\section{POLARIMETRIC SYSTEMS POTENTIALS AND RESTRICTIONS}

\section{A. Polarization parameters}

Polarization radars operate with different polarization of sounding waveform and/or received wave. They also can receive two orthogonally polarized waves. This allows to define distantly the next characteristics of hydrometeors: size, shape, spatial orientation and thermodynamical state. Polarization characteristics are described with scattering matrix that connects electrical field of reflected electromagnetic wave received by antenna with incident electrical field [13]. Some of the commonly used measured polarimetric variables that are obtained from parameters of scattering matrix are the next:

Radar reflectivity at horizontal polarization

$$
Z_{h}=\frac{4 \lambda^{4}}{\pi^{4}|K|^{2}}\left\langle\left|s_{h h}\right|^{2}\right\rangle
$$

Radar reflectivity at vertical polarization

$$
Z_{h}=\frac{4 \lambda^{4}}{\pi^{4}|K|^{2}}\left\langle\left|s_{v v}\right|^{2}\right\rangle
$$


Differential Refelectivity (DR)

$$
Z_{D R}=10 \log \frac{\left|s_{h h}\right|^{2}}{\left|s_{v v}\right|^{2}}
$$

Linead Depolarization Ratio (LDR)

$$
L D R_{h v}=10 \log \frac{\left|s_{h v}\right|^{2}}{\left|s_{v v}\right|^{2}} \text { or } L D R_{v h}=10 \log \frac{\left|s_{v h}\right|^{2}}{\left|s_{v v}\right|^{2}}
$$

Correlation Coefficient at zero shift

$$
\rho_{h v}(0)=\frac{\left\langle s_{v v} s_{h h}^{*}\right\rangle}{\left\langle\left|s_{h h}\right|^{2}\right\rangle^{\frac{1}{2}}\left\langle\left|s_{v v}\right|^{2}\right\rangle^{\frac{1}{2}}}
$$

Where the first index indicates the polarization of received electromagnetic wave and the second indes indicates the polarization of transmitte electromagnetic waves.

Polarimetric measurables allows us to take up the physical aspect of meteorological objects and obtain information about shape, orientation and physical state of hydrometeors. Nowadays the set of polarimetric parameters are used usually to solve the mentioned tasks.

\section{B. Shape, orientation and physical state of hydrometeors identification with polarimetry}

Very small particles are almost spherical. The spherical particles were considered with no polarization features per se. But the most precipitation is composed from nonspherical particles. The falling in stagnant air raindrops are oblate. The ice crystals are elongated and flattened. Thus the dual polarization radar measurements can help to identify hydrometeor shape and then make decision about their physical state.

The Differential Reflectivity (DR) is close to zero for spherical particles. It is different from zero in case of nonspherical particles as falling large raindrops. The Radar reflectivity at horizontal polarization also increases for the oblate falling hydrometeors. Therefore, it can help to discriminate rain (falling oblate hydrometeor) from the almost spherical small hail. Radar reflectivity will increase for large hail.

Differential Reflectivity is different from zero for nonsphercal particles. At the same time the Linear Depolarization Ratio increases with increasing hydrometeor nonsphericity. In case of chaotic orientation of nonspherical particles the radar reflectivity will be close to zero again. Correlation coefficient can be useful for identifying melting particles or mixed precipitation.

Thus to identify shape, orientation and physical state of hydrometeors successfully, the set of polarimetric parameters are required.

\section{Possibility to use polarimetryfor wind related phenomena identification}

Polarimetric parameters are sensitive to hydrometeor shape and their values depend on hydrometeor orientation. As it was shown above the polarimetric measurements allow to identify the physical state of hydrometeors and discriminate the liquid particles from the solid ones. It is important because the liquid hydrometeor orientation in turn depends on both wind direction and velocity [7]. Their shape is also dependent on other wind related phenomena including turbulence, downward updraughts, windshear. Therefore, one can conjecture that the polarimetric parameters allow to determine wind direction when changing the particle orientation under the influence of wind as well as intensity of wind related phenomena (including turbulence) as extent and character of drop deformation. This fact is important because the Doppler radars allows to calculate the radial component of hydrometeors motion only.

It is important to note as well that modern radars operate with average estimates and some important peculiarities of drop behavior caused by wind related phenomena can be veiled beyond the averaged estimates of reflections from hydrometeors assemble. Therefore, distinguishing hydrometeors with similar polarization properties inside the reflecting volume and estimating their differential impact into the polarization properties of reflected signal are important tasks for practical implementation of polarimetric methods for wind related phenomena detection and estimate.

\section{PRINCIPLE OF HYDROMETEORS DisCRIMINATION WITH Similar POLARIZATION PROPERTIES WITHIN THE RESOLUTION VOLUME}

In [14] the principle that allows to find amount or volume of the particles with similar polarization properties inside the reflecting volume is depicted.

The principle is based on measuring backscattering at different polarization angles. The measured backscattering is proportional to the number of particles $(N)$ in $1 \mathrm{~cm}^{3}$ and gives the value $\left(N a^{2}\right)_{i}$. Index $i$ represents polarization for which the measurements were done and $\alpha$ is tenzor that in common case can be represented as scattering matrix;

The number of particles usually is unknown that makes it impossible to define drops size using the intensity of backscattering only.

Next in [14] it is proposed to make measurement of refraction index at different polarization angle

$n=1+2 \pi \alpha N$.

Now it is possible to obtain the value $(N \alpha)_{i}$ at different polarizations.

where index $i$ again represents polarization of reflected electromagnetic oscillation. 
Combining two measurements, it is possible to obtain $N_{i}$ and $\alpha_{i}$ that were obtained at different polarization angles. Having information about $N$ and medium density the information about mass of single particles can be obtained [14]. Information about mass and structure of the particle allows to obtain the volume of particle that is also related to $\alpha$.

Taking into account the facts that the best reception of polarized signals is achieved when vectors of antenna polarization and signal polarization are coinciding we can say that the stronger the change of polarization, the weaker the energy of oscillations in the antenna tuned to receive oscillations with principal polarization; at the same time the higher energetic level of oscillations is received by the antenna tuned to polarization that is different from polarization of the sounding waveform.

Nature of liquid hydrometeors to change the polarization of reflected electromagnetic wave under the atmospheric dynamic phenomena influence allows us to conclude that maximum contribution into intensity of backscattering with some definite polarization is given by particles of correspondent size, shape and orientation. Following the presented principle of calculation number and mass of the particles in resolution volume it is possible to estimate the number of particles and their dimensions that contribute into backscattering at some definite polarization. Such estimation can be realized by measuring backscattering at different polarization angles. For this purpose, it is reasonable to use the radar system with numbers of receiving antennas that is larger than two (two antennas are commonly used nowadays in modern polarimetric radars: one receiving antenna can receive signals with main polarization - polarization of sounding waveform and another receiving antenna can receive signals with orthogonal polarization).

Such measurements allow to avoid averaging when polarization characteristics estimation and further loss of information about weight of contribution of separate liquid particle into final change of reflected electromagnetic wave polarization. Having this information, it is possible to distinguish number and dimensions of hydrometeors with similar polarization properties inside the reflecting volume.

Then, having obtained information about liquid hydrometeors with similar polarization properties that was obtained by radar antennas it is possible to calculate the numbers of particles and their dimensions. This information can be useful not only for dropsize distribution estimate and rainfall measurements but also for wind related phenomena detection and estimate with polarization methods. The description of radar systems for simultaneous measurement of backscattering at different polarization angles and obtaining socalled polarization spectrum [15] is presented in [16].

The approach to discriminate the hydrometeors with similar polarization properties is possible to demonstrate with the following diagram Fig.1.

Calculation of the volume of particle at different polarization angles requires the information about number of particles and medium density that is known and in turn related to $a$.

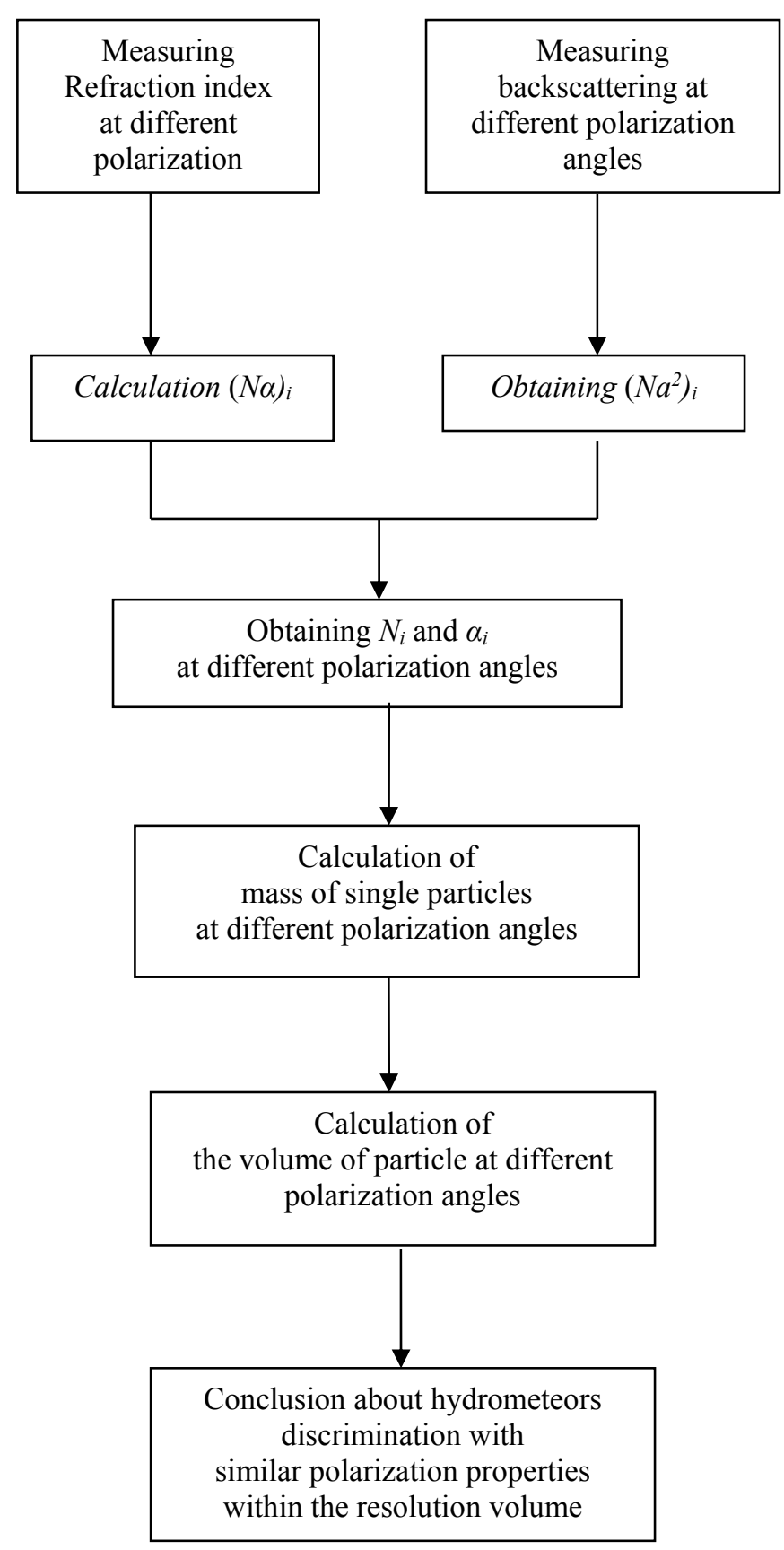

Fig.1. Steps of the process of hydrometeor discrimination with similar polarization properties

\section{POSSIBLE REALIZATION OF OF RADAR SYSTEM MEASURING POLARIZATION SPECTRUM}

Modern technologies allow to realize presented approach using the single printed antenna. Example of such antenna is shown in Fig. 2. 


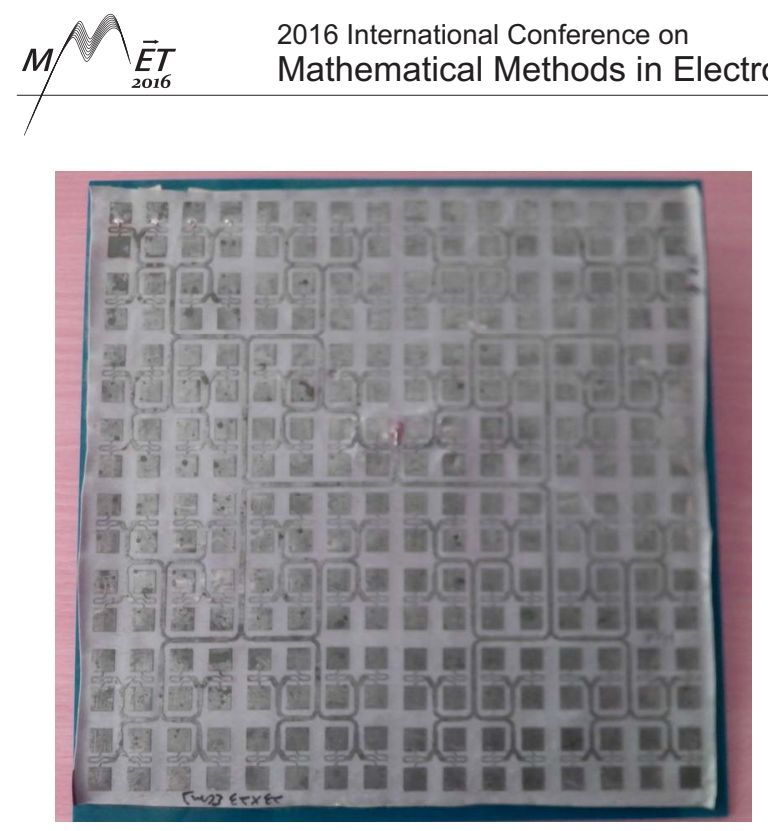

Fig.2. Example of Printed Antenna for Measuring Horizontally or Vertically Polarized Reflected Electromagnetic Wave.

The Power distribution system that is realized at this antenna allows to make measurements at vertical or horizontal polarizations. To measure energy of reflected from meteorological target electromagnetic wave at different polarization angles is possible by changing the antenna orientation.

For simultaneous measurement of reflected from meteorological targets electromagnetic wave at different polarization angles it is necessary to make additional correspondent power distribution system and make connection to the separate channels for further simultaneous processing. This realization requires the channel identity checking for reliable radar measurements.

\section{CONCLUSIONS}

In this paper we consider possibilities of radar polarimetry to solve different meteorological tasks including identification of atmospheric phenomena microstructure and hydrometeor orientation, hydrometeors size measurement, wind related phenomena estimation.

The approach to distinguish hydrometeors inside the reflecting volume with different polarization features was presented. The approach is based on information about hydrometeor size, shape and orientation.

The realization of the approach using the polarimetric system with multiple receiving antenna set that can receive signals of not only the two orthogonal polarizations but intermediate ones are shown.

The presented approach can allow to avoid averaging when polarization measurements and thus give posibility to make estimates of dynamic phenomena as well as to improve measurements for other tasks of radar meteorology including drop size distribution estimate, rainfall intensity estimate, wind speed measurement, strong wind shear detection.

\section{REFERENCES}

[1] F.J. Yanovsky, C.M.H. Unal, H.W.J. Russchenberg, L.P. Ligthart, "Doppler-polarimetric weather radar: Returns from wide spread precipitation," Telecommunications and Radio Engineering 66 (8), pp.715-72, 72007.

[2] F.J. Yanovsky, C.M.H. Unal, H.W.J. Russchenberg, L.P. Ligthart, "Doppler-polarimetric weather radar: Returns from wide spread precipitation," Telecommunications and Radio Engineering v. 66, issue 8, pp. 715-727, 2007.

[3] F.J. Yanovsky, H.W.J. Russchenberg, L.P. Ligthart, "DopplerPolarimetric models of microwave remote sensing of rain," (invited paper), Conference Proc. The 11th Conference on Microwave Technique (COMITE), pp. 47-62.

[4] F. J. Yanovsky, "Inferring microstructure and turbulence properties in rain through observations and simulations of signal spectra measured with Doppler-polarimetric radars," Book Chapter in: M.I. Mishchenko et al. (eds.), Polarimetric Detection, Characterization, and Remote Sensing, Springer, pp.501-542, 2011.

[5] F.J. Yanovsky, Simulation study of $10 \mathrm{GHz}$ radar backscattering from clouds and solution of the inverse problem of atmospheric turbulence measurements. Computation in Electromagnetics, IEE (UK) No. 420, 1996, pp. 188-193.

[6] F.J. Yanovsky, H.W.J. Russchenberg, C.M.H. Unal, "Retrieval of information about turbulence in rain by using Doppler-polarimetric Radar," Microwave Theory and Techniques, IEEE Transactions on $\mathrm{v}$. 53, no 2, pp. 444-450, 2005.

[7] Yu. A. Averyanova, "Use of Doppler-Polarimetric parameters for wind phenomena localization," Proceedings of the European Microwave Week, pp. 29-32, October 2004.

[8] Yu. Averyanova, A. Averyanov, F.J.Yanovsky, "Connection of Reflected Radar Signal with Liquid-Hydrometeor Deformation Rate," Proceedingsof the $3^{\mathrm{d}}$ Symposium on Microwaves, Radar and Remote Sensing, pp.217-219, August, 2011.

[9] Yu. Averyanova, A. Averyanov, F. Yanovsky, "The approach to estimating critical wind speed in liquid precipitation using radar polarimetry," Proc. of 14th International Conference on Mathematical Methods in Electromagnetic Theory, pp.517-520, August 2012.

[10] Yu. Averyanova, "Statistical Algorithm for Turbulence Detection using Polarization Features of Radar Reflections from Rain," Proc. of International Radar Symposium ( IRS 2015), pp. 593-596, June 2015.

[11] S.V. Buhman, "The experimental study of drops division," Vesnik of Academy of Science of Kazahskaya SSR, № 11, pp.38-43, 1954. (In Russian).

[12] A.S. Lyshevsky, "The movement of the liquid drops in the gaseous flow," Izvestiya Vuzov. Energetika, № 7. C. 75-81, 1963. (In Russian).

[13] Doviak, R.J., and D.S. Zrnic, "Doppler radar and weather observations" Academic Press, inc., 1993.

[14] H. C van de Hulst, "Light scattering by small particles," London, 1957.

[15] Yu. Averyanova, A. Averyanov, F.J.Yanovsky, "Polarization signal components estimate in weather radar," Proc. of 12th International Conference on Mathematical methods in electromagnetic theory, pp. 360-362, 2008.

[16] Yu. Averyanova, A. A. Averyanov, F.J. Yanovsky, "Polarimetric Radar,” Invention patent of Ukraine № u200804248, April 2008. (In Ukrainian). 